diskutiert werden. Der Bohrkopf ist am Rotablator distal mit Diamantsplittern besetzt. Bei der Rotation mit hoher Geschwindigkeit wird der Kalk in mikroskopisch kleine Partikel „verpulverisiert“. Im bohrkopfnahen Kalk können dabei Fissuren entstehen. Ein neues Verfahren sind Cutting und Scoring-Devices, die eine fokussierte Kraftübertragung über Klingen oder Scoring-Elemente (Drähte, Nitinolkäfig, Nylonfäden) ermöglichen.

\section{Nicht dilatierbar, was tun?}

Von einer nicht dilatierbaren Stenose spricht man, wenn sich der Ballon bei 20 bar nicht entfaltet. Man sieht das typische „dogboning“. „In solchen Situationen ist es nicht sinnvoll, einen größeren Ballon einzusetzen“, so Birkemeyer.
Vielmehr sollte der Plaque mithilfe des Bohrkopfs zunächst „modifiziert“ werden, bevor eine erneute Vordehnung mittels Non-Compliant(NC-)Ballon durchgeführt wird. Dann gelingt meist eine Stenting, es empfiehlt sich aber eine Hochdrucknachdilatation.

\section{Aorto-ostiale Läsionen}

Bei aorto-ostialen Läsionen besteht die Gefahr der Okklusion oder Dissektion durch den Führungskatheter. Auch die Stentimplantation ist oft inadäquat und es kann zu einem "geographical miss“, also zu einem Stenting distal des eigentlichen Ostiums oder zu einer zu weiten Protrusion in die Aorta kommen. Bei Repositionierung des Führungskatheters kann eine longitudinale Stentkompressi- on eintreten. Ist eine solche Stenose nach Vordehnung mit einem NC-Ballon dilatierbar, sollte bei einem deutlichen Recoil ein Cutting- oder Scoring-Ballon eingesetzt werden, bevor das Stenting mit einer Hochdrucknachdilatation erfolgt.

Zeigt sich nach einem primären Stenting einer eigentlich nicht dilatierbaren Stenose ein stark unterexpandierter Stent, bedeutet dies, dass die Revaskularisation nicht erfolgreich war. Diese Siuation geht mit einem hohen Risiko für eine Stentthrombose einher. Führt auch die Nachdilatation mit einem Druck bis 25 bar nicht zum Erfolg, sollte eine Bypass-Operation diskutiert werden. Ansonsten käme hier der Rotablator oder die Ultrahochdruckdilatation zum Einsatz. Dr.med. Peter Stiefelhagen

\title{
DAPT: Wann kürzer? Wann länger?
}

Die optimale Dauer einer dualen Plättchenhemmung (DAPT) nach Stentimplantation kann individuell unterschiedlich sein. Was sind die wichtigsten Faktoren in diesem Entscheidungsprozess? Dazu gab ein Experte Auskunft.

In der neuen ESC-Leitlinie zur dualen Plättchenhemmung beim akuten Koronarsyndrom wird ein stärker individualisiertes Vorgehen empfohlen“, so Prof. Ulf Landmesser aus Berlin. Die Standardempfehlung von zwölf Monaten gelte weiterhin und zwar unabhängig vom Stenttyp. Doch je nach Ischämierisiko und Blutungsrisiko sollte die duale Plättchenhemmung kürzer oder länger erfolgen.

Schon die Leitlinie von 2015 hat den vorrangigen Einsatz eines modernen P2Y12-Inhibitors wie Ticagrelor oder Prasugrel propagiert, wobei Prasugrel erst nach Kenntnis des Koronarbefundes gegeben werden sollte. Ticagrelor wird jetzt zusätzlich zu ASS empfohlen und zwar unabhängig von der initialen Behandlungsstrategie, also auch bei Patienten, die mit Clopidogrel vorbehandelt wurden. Die Umstellung sollte früh nach der Krankenhausaufnahme erfolgen und mit einer Loading-Dosis von $180 \mathrm{mg}$ beginnen (Klasse IB).

\section{Blutungsrisiko abschätzen}

Die Dauer der DAPT sollte sich am individuellen Ischämie- und Blutungsrisiko orientieren. Für die Beurteilung des Blutungsrisikos steht mit PRECISE-DAPT ein zuverlässiger Score zur Verfügung. In diese Risikobewertung fließen folgende Parameter ein: Hb-Wert, Alter, Nikotinabusus, Diabetes, Myokardinfarkt oder Zustand nach Infarkt, linksventrikuläre Auswurffraktion (LVED) $<30 \%$, Stentdiameter $<3 \mathrm{~mm}$, Paclitaxel beschichteter Stent und Venenbypass. „Ein Wert über 25 bedeutet ein deutlich erhöhtes Blutungsrisiko, sodass die DAPT schon nach sechs Monaten beendet werden sollte“, so Landmesser.

Umgekehrt sollte bei Infarktpatienten mit hohem Ischämierisiko die DAPT länger als zwölf Monate bis zu drei Jahren sein, allerdings nur dann, wenn die DAPT vorher ohne Blutungskomplikationen gut vertragen wurde.

Von einem erhöhten Ischämierisiko muss man ausgehen, wenn eines oder mehrere der folgenden Hochrisikomerkmale vorliegen: Alter $\geq 65$ Jahre, medikamentös behandlungsbedürftiger Diabetes mellitus, zwei Infarkte in der Vorgeschichte, koronare Mehrgefäßerkrankung, chronische Niereninsuffizi- enz mit geschätzter Kreatinin-Clearance $<60 \mathrm{ml} / \mathrm{min}$.

\section{Sonderfall Vorhofflimmern}

Besonders wichtig ist eine individuelle Risikoabschätzung, wenn bei gleichzeitig vorliegendem Vorhofflimmern zusätzlich eine orale Antikoagulation indiziert ist. Für solche Patienten wird als Standard eine Triple-Therapie mit ASS, Clopidogrel plus orales Antikoagulans für sechs Monate empfohlen (Klasse IIaB). Anschließend sollte die Therapie mit einem Plättchenhemmer -ASS oder Clopidogrel - über weitere sechs Monate fortgeführt werden. Danach ist bei stabilen Patienten nur noch das orale Antikoagulans notwendig. Doch bei erhöhtem Blutungsrisiko werde die TripleTherapie nur für ein Monat empfohlen.

In der Folge sollte nach Landmesser auf eine duale Therapie mit ASS oder Clopidogrel plus Antikoagulans umgestellt werden. Bei sehr hohem Blutungsrisiko sollte man entsprechend der Leitlinie ganz auf die Triple-Therapie verzichten und über zwölf Monate nur die Kombination Clopidogrel plus orales Antikoagulans geben (Klasse IIa A).

„Die neue Leitlinie ist ein wichtiger Schritt in Richtung individualisierte Therapie mit dem Ziel der Optimierung bei entsprechender Nutzen-Risiko-Abwägung“, resümierte Landmesser.

Dr. med. Peter Stiefelhagen 